I.

\title{
Untersuchungen über Blutgerinnung im lebenden Thiere und ihre Folgen.
}

\author{
Von \\ B. Naunyn \\ in Königsberg.
}

Die hier folgenden Mittheilungen schliessen sich an Beobachtungen an, die ich im Jahre 1868 bei Gelegenheit experimenteller Untersuchungen über Ieterus zufällig machte. Letztere sind bereits damals in der betreffenden Arbeit*) veröffentlicht. Im folgenden Jahre wurde das in diesen angeregte Thema durch Herrn Dr. Francken in Dorpat weiter bearbeitet; von ihm rührt ein bedeutender Theil der im Nachfolgenden mitzutheilenden Versuche her. Da Herr Francken weiterhin nicht in der Lage war, die begonnene Untersuchungsreihe fortzuführen, habe ich selbst den Gegenstand während meines Aufenthaltes in Bern aufgenommen. Ieh nehme um so weniger Anstand, im Einverständniss mit Herrn Francken die ron ihm in Dorpat unter meiner Leitung angestellten und damals in seiner Dissertation**) niedergelegten Versuche meiner jetzigen Darstellung einzuverleiben, als jene Dissertation in weiteren Kreisen vollkommen unbekannt geblieben zu sein scheint, wenigstens sind nur so die Einwendungen, welche in einer kïrzlich erschienenen Mittheilung***) gegen die Richtigkeit der von mir in

*) Beiträge zur Lehre vom Icterus. Reichert's u. Dubois' Archiv 1868

**) Ein Beitrag zur Lehre von der Blutgerinnung im lebenden Organismus and ihren Folgen. Inaugural-Dissertation. Dorpat 1870.

***) Schiffer, Angebliche Bitutgerinnung nach Injection freier fibrinoplastiseher Substanz (Orig.-Mitth.). Centralblatt für die medicinischen Wissenschaften 1872. Nr. 10. 
jener Arbeit 1868 veröffentlichten Beobachtungen erhoben wurden, begreiflich, wenn auch nicht berechtigt.

Man entzieht einem kleineren Thiere (Katze oder Kaninchen) einige Cubik-Centimeter Blut aus irgend einer Arterie oder Vene, und defibrinirt dasselbe vollständig durch Schlagen. Dann löst man die Blutkörperchen in dem defibrinirten Blute vollständig dadurch auf, dass man letzteres schnell hinter einander wiederholt gefrieren und wieder aufthauen lässt (Rollet). Man erwärmt das Blut auf 10 oder auch bis $30^{\circ} \mathrm{C}$. und befreit es mittelst langsamen Filtrirens durch feinstes Leinen von etwa noch nachträglich entstandenen Gerinnungen. Spritzt man von der so erhaltenen vollständig ,, lackfarbenen" Flissigkeit einige Cubikcentimeter (je nach der Grösse des Thieres $4,10-15$ ) unter den geeigneten Vorsichtsmaassregeln in eine grössere Vene ein, so folgt in der Regel sofort eine mehr minder reiche Gerinnung des Blutes in den centralwärts zum rechten Herzen führenden Venenstämmen, im rechten Herzen selbst und in der Pulmonalarterie. Die Folge ist selbstverständlich der augenblickliche asphyktische Tod des Thieres.

$\mathrm{Zu}$ empfehlen ist, dass man durch mikroskopische Untersuchung: des zu injicirenden Blutes controllire, ob die Auflösung der Blutkörperchen vollständig stattgehabt. Nicht selten finden sich auch in dem für das blosse Auge vollkommen lackfarben erscheinenden Blut zahlreiche, nicht gelöste Blutkörper bei mikroskopischer Besichtigung; dann ist selbstredend nochmaliges, oder sogar noch mehrfach wiederholtes Gefrieren nothwendig. Ausserdem scheint es für den Erfolg des Experimentes wesentlich zu sein, dass die injicirte Flïssigkeit sich in einem bestimmten Mengenverhältniss mit dem gleichzeitig die Vene durchströmenden Blute mische; ist der Injectionsstrom zu massenhaft, so drängt er wohl den Blutstrom ganz zurick, ist ersterer zu spärlich, so wird nicht die genigende Menge der Gerinnung erregenden Substanz dem Blute beigemischt.

In den weiter unten beschriebenen Versuchen wurde stets eine Spritze mit scharfer (s. g. Stich-)Canüle angewendet, deren Lumen wenig weiter war, als das der weiteren Stichcanulen an den gewöhnlichen Pravaz'schen Spritzen. Hiermit wurde die Injection in die frei präparirte Vene ohne jede vorgängige Unterbindung ausgeführt. Die Einspritzung geschah gleichmässig langsam in der Art, dass fuir jeden Cubikcentimeter je eine Minute verbraucht wurde. 
Versueh 1. Einem grossen Kaninchen werden 2 CCm. lackfarbenen*) Blutes in die Vena jugularis injicirt. Das Thier sofort asphyktisch, das Herz beim Oeffnen noch in unregelmässigen Contractionen, der rechte Ventrikel und Vorhof stark ausgedehnt von einem dunkelrothen, ihn vollständig erfullenden, zwischen die Trabekel verfilzten Gerinnsel. Das Gerinnsel setzt sich einerseits in die Arteria pulmonalis und deren Zweige, andererseits in die Vena cava superior und jugularis sinistra bis zur Einstichstelle fort, das Lumen der Gefässe prall ausfüllend. Die Jugularis dextra, ebenso wie die andern Körpervenen, enthält lediglich flüssiges Blut, das linke Herz ist leer.

Versuch 2. Grosses Kaninchen, nach der Injection sofort Dyspnöe, vor dem Tode das Herz noch zuckend eröffnet, zeigt genau denselben Befund.

Versuch 3. Starkes Kaninchen, $2 \mathrm{CCm}$. lackfarbenes Blut in gleicher Weise eingespritzt, genau derselbe Erfolg, das noch zuckend eröffnete Herz zeigt genau denselben Befund.

Versuch 4. Starkes Kaninchen, 11/2 CCm. lackfarbenes Blut, genau dasselbe Resultat, das Herz sofort nach der Injection unter tiefen Inspirationsbewegungen des Thieres untersucht.

Versuch 5. Kräftiges Kaninchen, 2 CCm. lackfarbenes Blat injicirt bewirken genau dasselbe.

Versuch 6. Kräftiges Kaninchen, $1 \mathrm{CCm}$. lackfarbenes Blut in 2 Minuten Dauer injicirt, genau das gleiche Resultat.

Versuch 7. Katze von $2000 \mathrm{Gramm}, 15 \mathrm{CCm}$. lackfarbenes Blut, sofort Dyspnöe, das linke Herz pulsirt stark bei der Eröffnung, ist leer, der rechte Vorhof enthält keine Spur von flüssigem Blut, nur ein festes, ihn weit ausdehnendes Gerinnsel, der rechte Ventrikel, neben vielem flitssigen Blut, ein gleiches Gerinnsel, das sich in die Pulmonalarterie und ihre Zweige, sie vollkommen ausfüllend, fortsetzt.

Versuch 8. Katze von ${ }^{`} 2000$ Gramm, $8 \mathrm{CCm}$. Blut, der gleiche Befund.

Versuch 9. Katze 2000 Gramm, der ganze rechte Ventrikel und die Pulmonalis ganz mit festem, zusammenhängendem Gerinnsel gefüllt.

Dass in den vorliegenden Versuchen die fraglichen Gerinnungen im rechten Herzen und den Gefässen der Thiere bei Lebzeiten der-

*) Mit dem Worte ,lackfarben" wird weiterhin das in der obigen Weise präparirte Blut bezeichnet. 
selben statt hatten, ist unzweifelhaft, da fast iiberall das Herz, soweit es nicht durch die pralle Anfüllung mit Gerinnseln daran gehindert war, sich bei der Eröffnung noch lebhaft contrahirte. Auch ist zweifellos unter solchen Umständen die Gerinnselbildung die Todesursache.

Es ist übrigens keineswegs nothwendig, dass das zur Injection benutzte lackfarben gemachte Blut von dem gleichen Thiere oder einem Thiere der gleichen Species stamme; indessen scheint die Energie, mit der das lackfarbene Blut das Blut im lebenden Thiere gerinnen macht, verschieden, je nach der Species, von der es stammt und bei der es angewandt wird. Genau auf diesen Punkt einzugehen, lag ausserhalb der Grenzen dieser Arbeit. Es darf auf das schon frïher iber die Verschiedenheiten der gerinnungserregenden Energie des Blutes verschiedener Thiere von Al. Schmidt*) Mitgetheilte verwiesen werden.

Versuch 10. Lackfarbenes Blut, ron einer andern Katze stammend, $4 \mathrm{CCm}$. einer 1500 Gramm schweren Katze injicirt, im rechten Herzen und der Arteria pulmonalis spärliche Gerinnsel.

Versuch 11. Katze von $3000 \mathrm{Gramm}, 20 \mathrm{CCm}$. lackfarbenes Kalbsblut; Gerinnsel im rechten Herzen und der Pulmonalis spärlich.

Versuch 12. Katze, $18 \mathrm{CCm}$. lackfarbenes Füllenblut; Gerinnsel im rechten Herzen und der Pulmonalis mässig reichlich.

Versueh 13. Katze, $20 \mathrm{CCm}$. lackfarbenes Hundeblut; das ganze rechte Herz und Arteria pulmonalis von festem, zusammenhängendem Gerinnsel prall ausgefültt.

Dass die Fähigkeit des lackfarben gemachten Blutes, Gerinnungen hervorzurufen jedenfalls zum Theil auf der stattgehabten Auflösung der Blutkörperchen ruht, ist ausser Frage, da defibrinirtes Blut ohne Auflösung der Blutkörperchen auch nach längerem Stehen in der Kälte oder Wärme eine solche Fähigkeit nicht besitzt, wie dies die zahlreich an Menschen wie an Thieren angestellten Versuche mit Transfusion beweisen. Es lag nahe, die Wirkung des Hauptbestandtheils der Blutkörperchen, des Hämoglobins, in dieser Richtung zu prüfen. Hatten doch die Untersuchungen Al. Schmidt's die Rolle, die dieser Körper oder die ihm sehr fest anhaftende fibrinoplastische Substanz bei der Blutgerinnung spielt, kennen gelehrt.

Zu den folgenden Versuchen wurde krystallinisches Hämoglobin benutzt, welches aus Hundeblut in der bekannten Weise dargestellt

*) Die bekannte Arbeit in Reichert u. Dubois' Archiv 1862. 
und durch einmaliges Umkrystallisiren gereinigt war. Einer weitern Probe auf seine Reinheit wurde der Körper nicht unterzogen. Lòsungen solchen Hämoglobins wirken in der That vollkommen wie lackfarben gemachtes Blut.

Versuch 14. Katze von $3000 \mathrm{Gramm}, 16 \mathrm{CCm}$. klarer, kalt gesättigter filtrirter Hämoglobinlösung. Sofortiger Tod, das noch pulsirende Herz enthält rechts, neben flüssigem Blut, zahlreiche Gerinnsel, die Arteria pulmonalis durch ein solches bis in alle Zweige vollständig gefüllt.

Versuch 15. Katze von $2500 \mathrm{Gramm}, 20 \mathrm{CCm}$. gleicher Hämoglobinlösung in die Jugularis injicirt, Befund genau wie bei Vers. 14.

Versuch 16. Katze von $2200 \mathrm{Gramm}, 12 \mathrm{CCm}$. Hämoglobinlösung in die Vena jugularis, Befund wie bei Vers. 14, nur Gerinnsel etwas spärlicher.

Von weiterem Interesse war zweifellos die Frage, ob die bis jetzt in Anwendung gebrachten Flïssigkeiten eine gleiche Wirksamkeit, wie in den grossen Körpervenen, dem rechten Herzen und der Pulmonalarterie, auch in andern Theilen des Kreislaufs entfalten würden. Die Möglichkeit lag vor, dass sowohl das in der Vena portarum, als das in den Arterien strömende Blut sich in dieser Beziehung anders verbielte. Gleichzeitig boten Versuche, in diesen Theilen des Kreislaufs in gleicher Weise Gerinnung zu erzeugen, die Aussicht dar, andere nicht uninteressante Fragen zu erledigen. Die Veränderungen, welche in den Organen des thierischen Körpers als einfache Folge der Blutgerinnung in den dieselben versorgenden Gefässen auftreten, sind noeh nicht völlig bekannt, da bis jeizt Mittel, solche Blutgerinnungen direct zu erzeugen, fehlten. Von den durch arterielle Embolie hervorgerufenen Veränderungen der Organe lässt es sich mit Sicherheit nicht behaupten, dass dabei lediglich die Aufhebung der Circulation in Betracht kommt. Jedenfalls besteht die Möglichkeit, dass die durch Gerinnung des Blutes in einem grösseren-arteriellen Gefässbezirke mit Einschluss seiner capillären Verbreitung bewirkte vollständige Aufhebung aller Circulation vollständig andere Erscheinungen hervorruft, als dies die einfach arterielle Embolie thut. Bei letzterer ist die Aufhebung der Circulation, wie dies Cohnheim's Untersuchungen*) zeigen, für den grössten Theil des Capillargefässsystems in dem von der embolisirten Arterie versorgten Bezirk stets nur eine voriubergehende; gerade der Wiedereintritt des, wenn auch verkehrt gerichteten Blut-

*) Untersuchungen ïber die embolischen Processe. Berlin 1872. 
stroms in die Capillaren ist es, der dort die prägnantesten Folgeerscheinungen, die Infarctbildung, hervorruft.

Von Einspritzungen in die Körpervenen konnten aber Kenntnisse der Folgeveränderungen in den Organen nicht gewonnen werden, weil hier stets die Gerinnung, wenn sie iiberhaupt statthat, zum schnellen Tode führt.

Was nun zunächst die Vena portarum anlangt, so findet auch in ihr sofortige Blutgerinnung nach Injection der geeigneten Flussigkeit in eine Mesenterialvene statt. Injection geschah auch hier in gleicher Weise mit der Stichcantile.

Versuch 17. Einem starken Kaninchen 2 CCm. lackfarbenes Blut nach Laparotomie in eine Dïnndarmmesenterialvene injicirt, Darm reponirt, Wunde vernäht, das Thier bleibt nach der Operation lebend, 1/2 Stunde nach der Operation das Abdomen des lebenden Thieres geöffnet; die Vena portarum im Stamme und in den Verzweigungen in der Leber von einem festen Gerinnsel vollkommen ausgefüllt.

Versuch 18. Katze von 2500 Gramm, Einspritzung von $13 \mathrm{CCm}$. lackfarbenen Bluts; 1 Stunde nach der Operation das Thier strangulirt, das Abdomen vor vollständigem Tode eröffnet; einer der Hauptäste der Vena portarum und seine Verzweigungen in der Leber durch Gerinnsel vollständig erfüllt.

In Fällen, wo das Thier die Blutgerinnung in der Vena portarum längere Zeit überlebt, zeigt das Gerinnsel die bekannten Veränderungen der auch auf andere Weise in Venen entstandenen Thromben. Das Gerinnsel entfärbt sich allmälig und nach einigen Tagen ist es der Innenfläche der Gefässwand adhärent geworden. Diese Erscheinungen sind ein weiterer Beweis für die Richtigkeit der alten Virchow'schen*) Ansicht, dass in vielen Fällen die Blutgerinnung das Primäre, die zu Adhärenz des Thrombus führende Veränderung der Venenwand das Secundäre sei.

Versuch 19. Katze von $1800 \mathrm{Gramm}, 10 \mathrm{CCm}$. Jackfarbenes Blut in eine Mesenterialvene; nach 4 Tagen der Tod; in der Leber gleich nach der Haupttheilung der Vena portarum ein scheckig verfärbter, theilweise adhärirender, 1 Zoll langer Thrombus, der das Lumen des Gefässes nicht völlig erfüllt.

Versuch 20. Katze von 2600 Gramm, 9 CCm. lackfarbenes Blut in eine Mesenterialvene; Tod am dritten Tage nach der Operation. In der Leber gleich nach der Theilung des Stammes der Vena portarum in einem Hauptaste ein zum Theil entfärbter, adhärenter

*) Thrombose und Embolie. Gesammelte Abhandlungen. Hamm 1862. 
Thrombus, der sich bis in ihre feinsten Verzweigungen verfolgen lässt. In dem zugehörigen Theile der Leber zahlreiche, stecknadelkopfgrosse, eircumscripte, scharf begrenzte, graulich verfärbte Herde, iu welchen das Mikroskop die Leberzellen verfettet, zum Theil fettig: zerfallen, sonst keine weiteren Veränderungen erkennen lässt.

Die Veränderungen, welche in der Leber 'des dem letzt angeführten Versuche unterlegenen Thieres bemerkt wurden, sind wenig ausgesprochen. Es scheint, dass in diesem Fall die Circulationsstörung durch die Blutgerinnung höchst unvollständig war; wahrscheinlich ist es, dass hier die Versorgung der Leber durch zwei von einander unabhängige Gefässe (Vena portarum und Arteria hepatica) in Betracht kommt; jedenfalls treten die entsprechenden Gewebsveränderungen viel deutlicher hervor, wenn die Gerinnung: in arteriellen Gefässen und ihren Capillarbezirken statthat.

Die Gerinnungserscheinungen in den arteriellen Gefässen und ihre Folgen wurden ausschliesslich an den Extremitäten von Katzen studirt. Dabei waren mancherlei Schwierigkeiten zu ïberwinden. Die Einspritzung mittelst der scharfen Canüle erwies sich als unausführbar: Die Injection des lackfarben gemachten Blutes in die in einen peripheren Arterienstumpf eingebundene 'Canüle war erfolglos. Es ist dies leicht begreiflich; denn es findet so die eingespritzte Flüssigkeit in den arteriellen Gefässen und Capillaren kein oder zu wenig Blut vor, um erhebliche Gerinnselbildung herbeifuhren zu können; es wurde nach vielen derartigen Injectionen lediglich in einigen kleineren Arterienästen Gerinnselbildung gefunden.

Um die Mischung der eingespritzten Flüssigkeit mit dem in der Arterie strömenden Blute zu bewerkstelligen, wurde folgendes Verfahren eingeschlagen. Es wurde eine T förmige Canüle angewendet, deren lotbrechter Schenkel sich nicht genau im rechten Winkel an die in einer Linie liegenden wagrechten Schenkel, sondern sehief so ansetzt, dass er mit dem einen dieser Schenkel einen spitzen, mit dem andern einen stumpfen Winkel bildet $(T)$. Diese Canüle wird in die Arterie so eingeschaltet, dass der stumpfe Winkel gegen die Peripherie, der spitze Winkel gegen den centralen Ursprung: gerichtet ist. Es ist diese Anordnung nothwendig, weil der durch die wagrechten Schenkel der Canüle nach ihrer Einbindung in die Arterie gehende Blutstrom obnehin ein sehr schwacher ist; derselbe wird dann, wenn nun die Injectionsflüssigkeit vom lothrechten Schenkel her eingespritzt wird, leicht vollständig zuriickgeworfen, oder wenigstens so geschwächt, dass die gewünschte Mischung der 
Injectionsflüssigkeit mit dem arteriellen Blute nicht erreicht wird. Auch bei Anwendung der $T$ förmigen Canüle mit schiefgeneigtem Schenkel ist die Vorsicht zu beobachten, dass die Injection langsam geschieht und ferner, dass jede Verschiebung der Caniile vermieden wird, weil diese leicht durch Zerrung der Arterie zur vorübergehenden Unterbrechung des Blutstromes führt.

Injection von lackfarben gemachtem, defibrinirtem Blut, in der angegebenen Weise in die Arterien bewerkstelligt, führte stets die umfangreichsten Thrombosirungen der peripherisch gelegenen Arterienstämme herbei; stets fand sich der Thrombus nach einigen Tagen adhärent, und niemals fehlten ausgebreitete und sehr eingreifende Veränderungen in den von den thrombosirten Arterien versorgten Geweben, den Muskeln. Die Abhängigkeit dieser Verånderungen von der Blutgerinnung ist vollkommen klar. Es traten dieselben in sehr verschiedenen Formen auf. Bald waren es einfach Herde vollständiger Anämie des Gewebes; welche sich in Folge der Arterienthrombose entwickelte, in anderen Fällen traten sehr umfangreiche Erweichungsherde auf. Die in letzteren enthaltenen breiartigen Massen bestehen zum Theil aus körniger, fettiger odel eiweissartiger Substanz, und sind aus Zerfall der Gewebsbestandtheile, besonders der Muskelfasern, hervorgegangen. Die mikroskopische Untersuchung zeigt mit Leichtigkeit die verschiedenen, namentlich durch Zenker bekannt gewordenen Formen der zerfallenden Muskelfaser, die "körnige" und "wachsartige" Degeneration.

Als Fäulnissprocesse im gewöhnlichen Sinne sind die Vorgänge, welche zu diesem Zerfall fithrten, nicht zu bezeichnen, denn die beschriebenen Erwejchungsproducte zeigten niemals erheblich widerlichen Geruch. Auch Formen, die als Pilze hätten angesprochen werden können, wurden darin nie beobachtet. Dennoch scheinen diese Erweichungsherde einen sehr deletären Einfluss auf das Leben der Thiere ausgeübt zu haben, der wohl nur auf die Aufnahme von Zersetzungsproducten aus denselben zurückgeführt werden kann. Diese Thatsache ist nicht ohne Interesse, da sie mit Sicherheit erweisen würde, dass auch Producte von solchen Zersetzungsprocessen im Organismus, welche (soweit vorläufig wenigstens zu übersehen) nicht durch Einfiihrung s. g. Fäulnisserreger hervorgerufen "sind, starke vergiftende Eigenschaften besitzen; eine Anschauung, die mit vielen längst bekannten Beobachtungen der Pathologen in vollkommenem Einklange steht. Dass übrigens zufällig den gerinnungserregenden Injectionsflüssigkeiten beigemengte s. g. septische Keime 
mit eingeftihrt wurden, ist natürlich mit Sicherheit nicht auszuschliessen. Dass auf der Wirkung solcher die hier auftretende Zersetzung beruht, ist jedenfalls sehr unwahrscheinlich. Eiterbildungen wurden in den betreffenden Herden nur selten (2 Mal) bemerkt. Was die Entstehung der Erweichungsherde anlangt, so entwickelten sich dieselben zum Theil unter dem Bilde des s. g. hämorrhagischen Infarctes; in anderen Fällen fehlten alle umfangreicheren Blutungen in die später unter Erweichung zerfallenden Gewebe vollkommen. Es sind diese Unterschiede leicht zu begreifen; dass und wie Infarctbildung zu Stande kommen muss, wenn in einer "Endarterie“ thrombotischer Verschluss stathat, ist nach Cohnheim's Untersuchungen vollkommen klar. Ebenso klar ist es, dass jener Vorgang ausbleiben muss, wenn, wie es in mehreren der Beobachtungen der Fall war, sich die Gerinnung nicht nur auf die Arterienstämmchen, sondern durch die Capillargefässe hindurch sich sogar bis zu den abführenden Venenstämmen erstreckt. Einige Male fand die Gerinnung unter dem Einfluss der in die Arterien eingespritzten Flussigkeiten iberhaupt erst jenseits der Capillaren in den Venenstämmen statt. Dann verlief der Vorgang wie bei directer Injection in letztere selbst, d. h. es setzte sich die Gerinnung bis zum rechten Herzen und in die Lungenarterie fort und fubrte so zum sofortigen Tode. Die Ursache der ausbleibenden Gerinnung in den Arterien ist in solchen Fällen am einfachsten so zu erklären, dass in Folge von Zerrung an der. Cantile während der Injection der Blutstrom unterbrochen oder wenigstens so weit geschwächt war, dass die Gerinnung verursachende Injectionsflisssigkeit die nöthige Menge des gerinnungsfähigen Blutes nicht in den Arterien und den Capillaren, sondern erst weiterhin in den Körpervenen vorfand.

Versuch 21. Katze von $3000 \mathrm{Gramm}, 16 \mathrm{CCm}$. lackfarbenes Blut in die linke Cruralis; folgende Tage schnell zunehmende Sehwäche, nach 2 Tagen das sehr !elende Thier getödtet, an der Innenfläche des linken Oberschenkels fast wallnussgrosser Erweichungsherd, der einen grossen Theil der Adductoren einnimmt, von vollkommen breiiger, nicht stinkender Masse erfüllt. Das umgebende Gewebe, namentlich der obere Theil der Adductoren, stark blutig infiltrirt. Ein ähnlicher, kirschkerngrosser Herd im innern Kopf des Musculus gastrocnemius. In der Arteria cruralis gleich unterhalb der Injectionsștelle ein scheckig entfärbter, leicht adhärirender, das Lumen vollständig ausfüllender Thrombus, der sich etwas bis über die Theilungstheile in der Kniekehle hinaus fortsetzt. Alle von der thrombosirten Arterie abgehenden Muskeläste 
und namentlich ein solcher auf den grossen Erweichungsherd hin und durch denselben hindurchziehender Ast vollkommen durch feste, zusammenhängende, leicht adhärirende Gerinnsel verschlossen. Ueberall sind auch die die thrombosirten Arterien begleitenden Venen in gleicher Weise thrombosirt. Der Inhalt der Erweichungsherde besteht zum grossen Theil aus feinkörnigen, nur zum Theil in Essigsäure löslichen Massen; dazwischen finden sich zahlreiche Trïmmer zerfallender Muskelfasern, nirgends, auch nicht an der Grenze gegen das gesunde Gewebe, Anhäufung von Eiterkörpern.

Yersuch 22. Katze von $2500 \mathrm{Gramm}$, in die Arteria cruralis dextra $18 \mathrm{CCm}$. lackfarbenes Katzenblut. Die Extremität wird nicht wieder beweglich, am folgenden Tage stark ödematös. Nach 48 Stunden verendet das Thier. Section 3 Stunden nach dem Tode. Im Heirz- und Venènblut zahlreiche Gasblasen. Die rechte Hinterextremität stark ödematös. Stamm der Cruralis von der Injectionsstelle ab von leicht adhärirendem Thrombus erftullt, der sich auch in die Art. tib. ant. und in zahlreiche von der Cruralis abgehende Muskeläste fortsetzt. Die Venen enthalten nur fliussiges Blut. Im oberen Theil des Extens. halluc. long. und Umgegend haselnussgrosser Erweichungsherd, von vollkommen zerfliesslicher Masse erfült. Ein eben solcher kleinerer, weniger stark erweichter Herd im äusseren Kopf des Muse. gastrocnemins. In den Muskeln des Oberschenkels zahlreiche Herde von Bluterguss in die Muskelsubstanz von Stecknadelknopf- bis Wallnussgrösse. Mikroskopische Untersuchung zeigt die Inbaltsmassen der Erweichungsherde ganz gleich wie im vorigen Versuch. In den Erweichungsherden wie in den Infareten die Capillaren durch Blutgerinnsel stark ausgedehnt und häufig' zerrissen.

Versuch 23. Katze von 2800 Gramm, 16 CCm. gesättigter Hämoglobinlösung in die Art. crur. dextra; unter schnell zunehmender Schwäche der Tod nach 24 Stunden. Section 12 Stunden nach dem Tode. Im Blut des Herzens und der grossen Venenstämme zahlreiche Gasblasen; rechte Hinterextremität stark ödematös geschwellt. Von der Injectionsstelle abwärts gleichmässig dunkel gefärbter, kaum adhärirender Thrombus, der die Art. erural. vollständig ausfüllt, sich in alle von ihr abgehenden Muskeläste, nur auf eine kurze Strecke in die Tib. ant, auf eine längere in die Tib. post. fortsetzt. Die Venen enthalten nur fiusssiges Blut. Die unteren Partien der Adductoren, wohin zahlreiche thrombosirte Gefässe führen, bereits vollständig erweicht. Die Inhaltsmassen der Er- 
Untersuchungen uiber Blatgerinnung im lebenden Thiere u. ihre Folgen. 11

weichungsherde wie in den vorhergehenden Versuchen. In den iibrigen Muskeln massenhafte Infarete von verschiedener Grösse.

Versuch 24. Kater von $2200 \mathrm{Gramm}, 15 \mathrm{CCm}$. lackfarbenes Blut in die linke Cruralis;; sofort asphyktischer Tod; das pulsirende Herz links vollkommen leer, rechts sehr starke Gerinnsel, die sich in die Pulmonalis, diese vollständig ausfiillend, fortsetzen etc. Die Arterien und Venen der zur Injection benutzten Extremität frei.

Versuch 25. Katze von 3100 Gramm, in die rechte Cruralis $13 \mathrm{CCm}$. lackfarbenes Blut; sofort asphyktischer Tod. Vena cava ascend. im oberen Theile starkes Gerinnsel, welches den rechten Vorhof prall ausfüllt. Der rechte Ventrikel neben Gerinnsel noch etwas flüssiges Blut. Der linke Ventrikel nur flüssiges Blut. Ebenso die Arterien und Venen der rechten Hinterextremität.

Versuch 26. Katze von $2200 \mathrm{Gramm}, 11 \mathrm{CCm}$. lackfarbenes Blut in die rechte Carotis, sofortiger Tod; im rechten Herzen die schon oft beschriebenen Gerinnsel. Carotides und Jugulares frei.

Es wäre gewiss nicht ohne Interesse gewesen, die Folgen der Blutgerinnungen in den arteriellen Gefässen auch noch an anderen Organen zu verfolgen. Indessen ist nicht zu erwarten, dass sich dieselben dort anders gestalten, wie die hier geschilderten in den Weichtheilen der Extremitäten; ausserdem zeigte sich, dass bei dergleichen Injectionen in die Carotis hier wenigstens in einer Anzahl von Versuchen Gerinnungen inberhaupt nicht eintreten. Es wurde daher der Gegenstand in dieser Richtung nicht weiter verfolgt.

Sichere Grinde für das häufige Ausbleiben der Gerinnung in diesem Gefässgebiet anzugeben ist nicht möglich, doch bedarf es wohl keiner weiteren Auseinandersetzung, dass dasselbe nicht geeignet ist, gegen die Eindeutigkeit der vorher mitgetheilten Versuchsresultate geltend gemacht zu werden.

Von besonderem Interesse nun erschien die Frage, ob die Lösung der Blutkörperchen innerhalb der Gefässbahn selbst in gleicher Weise zur Gerinnselbildung führt, wie Injection von lackfarbenem Blut oder von Hämoglobinlösung. Es ist die Frage nach den Angaben von Joh. Ranke*) bereits im positiven Sinne entschieden. Derselbe erklärt, dass die bekannte tödtliche Wirkung der gallensauren Salze in grösseren Dosen auf ihrer Fähigkeit, die Blutkörperchen zu lösen, beruhe, da, ,wie Naunyn frïher nachgewiesen, Blutkörperchenlösung, in die Venen eingespritzt, Gerinnung herbeiführt." Ranke fübrt eine Anzahl von Versuchen an, in welchen

क) Die Blutyertheilung and der Thätigkeitswechsel der Organe. Leipzig 1871. 
er nach Injection gallensaurer Salze in die Körpervenen Thromben in dem rechten Herzen und der Pulmonalis als Todesursache fand.

In jener Allgemeinheit scheint indessen seine Behauptung nicht vollkommen haltbar, zunächst da Gallensäuren die gleiche Wirkung: auch bei Einspritzung in die Arterien entfalten und hier jedenfalls die Gerinnung als Todesursache nicht nachgewiesen ist.

In einigen Fällen nun von Injection gallensaurer Salze, die nach der Ranke'schen Angabe vorgenommen wurden, trat der Tod ein, ohne dass in irgend einem Gefässe Thromben nachzuweisen gewesen wären. Auch das rechte Herz und die Pulmonalis enthielten lediglich flüssiges Blut.

Dennoch sind die Angaben von Ranke in so weit richtig, als, wie mich schon früher vorgenommene Versuche gelehrt hatten, die durch gallensaure Salze herbeigeführte Auflösung der rothen Blutkörperchen im Blute dort Gerimnung herbeiführt. Nur ist allerdings der Beweis hier schwieriger zu liefern, weil die Resultate hier: weniger constante sind, als in den Versuchen mit Injection der Blutkörperchenlösung selbst. Denn es müssen hier offenbar viele Bedingungen, die nicht alle in die Hand des Experimentators gegeben sind, erfüllt sein, damit die gewünsehte Wirkung eintritt. Einmal muss die eingespritzte Gallensäurelösung sich mit dem Blute grade in der zur schnellen und ausgiebigen Lösung der Blutkörperchen nöthigen Weise mischen, dann darf wieder die so entstehende Blutkörperchenlösung nicht zu sehr durch die injicirte Gallensäurelösung verdünnt werden, sonst fehlt die gerinnungsfähige Substanz. Es kann daher nicht Wunder nehmen, wenn manche Injection von Gallensäurelösung sogar in tödtlicher Dosis in dieser Richtung kein positives Resultat erzeugt; es beweist dies nur, dass die tödtliche Wirkung der Gallensäuren nicht allein auf ihrer Fähigkeit, die Blutkörperchen zu lösen und dadurch Gerinnungen zu machen, beruht.

In nachfolgenden Versuchen wurde der Injection in eine Körpervene die Einspritzung in eine Mesenterialvene vorgezogen. Bekanntlich ertragen die Thiere die Einfihrung der Gallensäure auf diesem Wege weit besser. Man konnte hoffen, die Folgen der Blutkörperchen-auflösenden Wirkung der Gallensäure besser zu beobachten, weil hier grössere Dosen erlaubt sind.

Ausserdem war voraussichtlich der Nachweis von Thrombosenbildung in kleineren, vielleicht schon capillären Verzweigungen des zur Injection benutzten Gefässes (aus dem Nachweis der Folgeveränderungen des betreffenden Organs, hier der Leber) leichter zu 
Uṇtersuchungen tiber Blutgerinnung im lebenden Thiere u. ihre Folgen. 13

führen, wenigstens war ich von früheren Versuchen her in dieser Richtung mit letzterem Organ besser vertraut.

Um etwa in kleineren oder capillären Gefässen bei der Injection der gallensauren Salze auftretende Thrombosen als gleich damals entstanden erkennen zu können, wurden der Injectionsflüssigkeit Farbstoffpartikelchen in geringer Menge beigemischt. Die Thiere wurden dann nach der Injection (sofern sie dieselbe ïberstanden) mehrere Tage am Leben belassen. Fanden sich jetzt in den mikroskopisch in der Leber nachzuweisenden Thromben die betreffenden Farbstoffkörnchen vor, so ist darnach gewiss, dass dieselben gleich bei der Injection entstanden sind; es ist dies um so mehr gewiss, als in solchen Lebern die von den thrombosirten Gefässzweigen versorgten Partien ganz circumscript, in höchst charakteristischer Weise verändert, wiederum erweicht gefunden wurden. In einem Fall entstand sofort bei der Injection gleichsam unter den Augen des Beobachters noch zu Lebzeiten des Kaninchens ein massenhaftes, die ganze Vena portarum mit ihren Zweigen in der Leber ausfïllendes Gerinnsel.

Versuch 27. Kräftiges Kaninchen, in eine Vena meseraica $4 \mathrm{CCm}$. $20 \%$ Lösung von gallensauren Salzen. Nach drei Minuten beginnende Convulsionen. Sofort wird das Abdomen, und während das Thier noch athmet, der Stamm der Vena portarum eröffnet. Derselbe ist vollständig erfüllt von festem, dunkelrothem Thrombus, welcher sich einerseits in der zur Injection benutzten Vena meseraica, andrerseits in allen Verzweigungen der. Vena portarum in der Leber bis in die feinsten Verzweigungen fortsetzt in gleicher Vollständigkeit und Festigkeit.

Versuch 28. Starkes Kaninchen, gleiche Gallensäurelösung mit Zinnober gemischt, $3 \mathrm{CCm}$. in eine Vena mesaraica. Während der Injection Unruhe. Das Thier nach der Operation vollkommen munter. Am dritten Tage nach der Operation Tod. Section etwa 8 Stunden nach dem Tode. In der Leber zahlreiche stecknadelknopf- bis mehr als kirschkerngrosse Herde, innerhalb welcher die Lebersubstanz weich, weiss gefärbt; an der Peripherie mit intensiv roth gefärbtem Saum gegen das übrige stark ikterisch gefärbte Lebergewebe scharf abgegrenzt. Auf Durchschnitten die feinsten Aeste der V. portarum in den Herden von zinnoberroth gefärbten Thromben erfüllt.

Bei der mikroskopischen Untersuchung in den Herden die Leberzellen stark verfettet. An der Peripherie die Capillaren stark ausgedehnt, in ihnen und in ihrer Umgebung zahlreiche rothe Blutkörperchen. Die Thromben in den zuführenden Portalästen, fast 
vollständig entfärbt, enthalten massenhaften Zinnober, wie namentlich Längsschnitte zeigen. Nirgends im Gewebe Kernanhäufungen.

Versuch 29. Starkes Kaninchen, $3 \mathrm{CCm}$. gallensaure Salze in die $V$. meser. Thier nach der Operation vollkommen munter. Am 4. Tage getödtet. Sectionsbefund genau wie irm vorigen Versuch, nur kein Ioterus der Leber.

Dass die Blutgerinnung; wie sie im Gefolge der Injection von Lösungen gallensaurer Salze hier auftrat, in der That die Folge der durch letztere bewirkten Auflösung der rothen Blutkörperchen ist, scheint mindestens sehr wahrscheinlich, da andre Substanzen, die in gleicher Weise die Blutkörperchen lösen, auch in gleicher Weise Blutgerinnung herbeiführen, wenigstens geben Aethereinspritzungen genau dasselbe Resultat. Dass es sich übrigens in beiden Fällen nicht etwa um eine solche Eiweissgerinnung und dadurch bedingte Verstopfung von Gefässen handelt, beweist die bekannte Thatsache, dass weder Gallensäurenlösung noch Aether in geringer Menge in defibrinirtem Blute Niederschläge hervorbringen.

Versuch 30. Starkes Kaninchen. Injection von etwa $3 \mathrm{CCm}$. Aether in V. mesaraica. Das Thier elend. Drei Stunden nach der Operation vivisecirt. Ein Hauptast der Vena port. in der Leber und seine Zweige ron festem, dunkelrothem Thrombus ganz erfüllt.

Versuch 31. Mittleres Kaninchen. Einige CCm. Aether mit etwas frisch gefälltem Berlinerblau gemischt. Kaninchen nach überstandener Narkose vollkommen munter, nach 4 Tagen getödtet. Die Leber zeigt ganz dieselben Veränderungen wie oben in den Gallensäureversuchen beschrieben. In den Thromben der zu den Erweichungsherden fithrenden Portaläste blaues Pigment nachweislich.

Ich habe bis jetzt vollständig unterlassen die mitgetheilten Thatsachen für die Beurtheilung der Lehre von der Blutgerinnung zu verwerthen. Es geschah dies hauptsächlich, weil ich in der ganzen Zeit, während welcher ich mich mit den hier mitgetheilten Untersuchungen beschäftigte (lediglich Experiment 28 u. 30 ist später als Juli 1871 angestellt) dem Glauben lebte, die Deutung dieser Thatsachen sei zweifellos. In der Annahme, dass die ältere von AI. Schmidt (l. c.) entwickelte Anschaung bereits auf sicherster Basis begründet sei, meinte ich, dass dieselbe in den Resultaten der hier mitgetheilten Versuche lediglich eire weitere Stütze fände. Die in den Blutkörperchen in reichlicher Menge vorfindliche, aus dem Hämoglobin entstehende oder ihm fest anhaftende fibrinoplastische Sub- 
Untersuchungen über Blutgerinnung im lebenden Thiere u. ihre Folgen. 15

stanz ist es, so besagte die Lehre, die sich unter jenes Forschers Aegide entwickelt hatte, welehe zusammentretend mit der im Blutserum stets im Ueberfluss vorhandenen fibrinogenen Substanz die Fibrinbildung und mit ihr die Blntgerinnung hervorruft; so liess sich der Erfolg der Einspritzungen gelöster Blutkörper (Hämoglobinlösung) und der Lösung der Blutkörper im kreisenden Blute erklären. Auch warum im lebenden Blute in der Norm Gerinnung nicht eintritt, war danach leicht begreiflich: es bedarf in der Fliissigkeit der Gegenwart einer gewissen Menge beider fibrinbildender Substanzen, damit es zur Gerinnung komme. Zu der nöthigen Aufhäufung der fibrinoplastischen Substanz kommt es aber nicht im Serum des kreisenden Blutes, weil einerseits stets nur wenig davon aus den Körperchen ins Serum übertritt, andrerseits die wirklich im Serum vorhandene Menge davon, wie alle eiweissartigen Körper, im Blute der fortdauernden Zersetzung unterliegt.

Für das Ausbleiben der Gerinnung im lebenden Körper fand Al. Schmidt (vgl. die eben erschienenen Hefto von Pflugers Archiv) jetzt einen andern Grund. Zum Zustandekommen der Gerinnung genügt nicht das Vorhandensein beider fibrinbildender Substanzen, sondern es bedarf dazu des Hinzutretens eines Fermentes, welches unter normalen Bedingungen jedenfalls in dem im lebenden Körper kreisenden Blute nicht vorhanden ist. Das Hämoglobin aber wirkt lediglich beschleunigend auf die Gerinnung. Diese Wirkung desselben ist eine reine Contactwirkung und kommt nur dem Hämoglobin zu, wie es sich in den Blutkörperchen vorfindet. Dieses übt die Contactwirkung aus, auch wenn es in den rothen Blutkörperchen eingeschlossen ist. Reines krystallisirtes Hämoglobin entbehrt, wie der Contactwirkungen iberhaupt, so auch der hier in Betracht kommenden vollständig, und auch Ueberfïhrung des in den Blutkörperchen vorhandenen Hämoglobin in das Serum durch irgend welche Lösung der rothen Blutkörperchen raubt dem Hämoglobin schnell, namentlich bei irgend längerer Berührung mit atmosphärischer Luft, jene eigenthümliche (Contact-) Wirkung.

Mit dieser neueren Anschauung des um die Lehre von der Blutgerinnung so hoch verdienten Forschers sind die hier mitgetheilten Thatsachen schwer in Uebereinstimmung zu bringen. Was die Versuche mit Einspritzung von Blutkörperchenlösung anlangt, so ist allerdings leicht begreiflich, dass bei der zur Bereitung des lackfarbenen Blutes eben so wie der des Hämoglobin nöthigen Behandlung des Blutes das Sehmidt'sche Ferment entstehen muss; und auch bei Injection von Lösung einmal umkrystallisirten Hämoglobins 
könnte immer noch Beimengung des schwer von letzterer Substanz zu trennenden Fermentes in Betracht kommen.

Doch genügt die einfache Injection dieses Fermentes ins kreisende Blut nicht, um im letzteren Gerinnung zu erzeugen. Dies beweist die Thatsache, dass die Transfusion lediglich defibrinirten Blutes, welches nach Schmidt stets reichlich Ferment enthält, eine solche Wirkung nicht ausübt.

Noch weniger vereinbar mit der nenen Schmidt'schen Lehre ist aber die Thatsache, dass auch die Lösung der rothen Blutkörperchen (durch Aether oder gallensaure Salze) Gerinnung herbeiführt. Zur Erklärung dieser Thatsache müsste man entweder annehmen, dass gerade die Lösung der Blutkörperchen durch die eingeführten Substanzen zum Auftreten der Fermentwirkung führt oder aber dass doch auch im Blute, welches im lebenden Organismus kreist, stets gering'e Mengen des fraglichen Fermentes vorhanden sind, welche nur wegen Mangel der die Gerinnung beschleunigenden Substanz nicht zur Wirkung kommen.

Die Annahme, dass geringe Mengen des fraglichen Fermentes auch im kreisenden Blute vorhanden sind, ist trotz $\mathrm{Schmidt}$ 's Mittheilungen nicht unstatthaft. Solch' geringe Mengen konnten ihm bei den Methoden, welche er zum Nachweis desselben benutzte (Fällung: des Fermentes durch Alkohol etc.), leicht entgehen; ist es doch bekannt, dass fermentartige Körper bei solchen Fällungen leicht einen Theil ihrer Wirksamkeit verlieren. Andererseits darf vielleicht für die Entstehung des Fermentes hier im Moment der Lösung der rothen Blutkörperchen die neuerdings von Tiegel*) mitgetheilte Beobachtung herangezogen werden. Dieser Forscher fand, dass das Blut seine fermentirende Wirkung auf Dextrin (Glykogen) nur im Momente der Auflösung der rothen Blutkörperchen ausïbt.

Doch genügt, wie schon oben gezeigt, das Auftreten des Fermentes im Blute nicht, um dort Gerinnung herbeizuführen; hierzu bedarf es jedenfalls noch des Hinzutretens einer Gerinnung-beschleunigend wirkenden Substanz. In den hier mitgetheilten Versuchen weist Alles auf das ins Blutserum gelangende Hämoglobin als solehe hin; doch ist es klar, dass auch in dieser Riehtung eine Uebereinstimmung zwischen den meinigen und Schmidt's Versuchen nicht besteht; denn des letzteren Forschers Angaben lauten dahin, dass die Gerinnung-beschleunigende Wirkung des Hämoglobins fast ausschliesslich dem in den Blutkörperchen eingeschlossenen zukommt.

*) Pflüger's Archiv 1872. 
Untersuchungen tuber Blutgerinnung im lebenden Thiere u. ihre Folgen. 17

Die Wirksamkeit desselben nimmt nach Sehmidt schon mit der Auflösung der es einschliessenden Blutkörperchen enorm ab und fehlt dem krystallinischen bereits vollkommen - in unseren Versuchen zeigt sich lediglich das durch Lösung der Blutkörper aus denselben frei gemachte und das krystallisirte Hämoglobin wirksam und beides gleichmässig wirksam.

Schliesslich sei noch erwähnt, dass Blutkörperchenlösungen, wie sie zu Injectionen in den beschriebenen Versuchen angewandt wurden, schädliche Wirkungen auf die Organe des lebenden Thieres entfalten, auch da, wo sie nicht Gerinnungen herbeiführen. Wenigstens zeigten öfters die derartig behandelten Thiere schwere Erscheinungen, ja es erfolgte einige wenige Male der Tod, ohne dass nachweisbare Gerinnungen stattgehabt hatten. Namentlich trat dies bei Injection derartiger Flüssigkeit in die Carotiden hervor, in welchen, wie vorn erwähnt, Gerinnsel niemals zur Beobachtung kamen. Eine Erklärung dafür zu geben, ist unmöglich; vielleicht dass das Hämoglobin als Sauerstoffäuber in den Geweben wirkt, vielleicht dass auch der Beobachtung sich entziehende capilläre Gerinnungen in Betracht kommen; hier geschieht der Thatsache lediglich deshalb Erwähnung, damit nicht andre Arbeiter (wie Schiffer 1. c.) glauben möchten, dass diese Wabrnehmung uns entgangen soi.

Königsberg im November 1872. 